\title{
El directivo organizacional como verdadero líder
}

\section{Resumen}

Las organizaciones modernas requieren a directivos que orienten al equipo de trabajo, que sean motivadores y facilitadores de las labores de los colaboradores, pues según el grado como se sientan acompañados, así será su productividad. Una de las grandes motivaciones de los individuos es tener a un jefe que los apoye, los asesore, los conozca, se interese por sus asuntos y los haga sentirse plenamente respaldados en cualquier circunstancia.

Pero, ¿y cómo es en la realidad? ¿Qué tan fácil es actuar como líder? Podría parecer, según se infiere de cuanto comentan los funcionarios, que en la mayoría de las empresas el liderazgo es bastante escaso; a menudo, en las conversaciones informales entre subalternos se oyen comentarios en el sentido de que "Verdaderamente, no hay líderes, sino jefes.'Y es claro que si un jefe no cuenta con unas características especiales de líder, está lejos de serlo.

Palabras clave: Liderazgo, motivación, trabajo en equipo, directivo, jefe.

\section{Abstract}

The modern organizations required executives who orientate the work team, who are motivating, who make more easy the labors of their collaborators, because in the grade they feel more accompanied, it will be reflect in their productivity. One of the biggest motivations of the individuals is having a boss that supports them, advise them, know them, a boss that is interested in their matters and they feel fully supported in any circumstance.

But how is it really? How much easier is act like a leader, it seems for the comments of the employees, than in majority of enterprises leadership is really limited; often in informal conversations between subordinates you heard comments like "really there are no leaders, but bosses" and it's very clear, that if a boss doesn't have special characteristics of leadership he is beyond of been a leader.

Key words: Leadership, motivation, work in team, executive, boss.

Recibido: 16 de abril de 2012

Aceptado: 27 de julio de 2012

\section{Introducción}

El liderazgo es un concepto que en el medio organizacional ha venido tomando fuerza durante los últimos años. Muy a menudo se habla de liderazgo estratégico, de coaching ${ }^{2}$, de líderes comunitarios, de líderes sociales, de empresas líderes en el mercado, y hasta de líderes negativos. En las organizaciones los directivos desean ser líderes; sin embargo, en la mayoría de los casos los jefes no poseen los atributos que identifican al líder.

Con este escrito se desea manifestar cuáles son las características que debe poseer un directivo para ser reconocido como líder, qué condiciones personales y profesionales se requieren para ejercer un liderazgo efectivo y cuán fácil es para los jefes en las organizaciones lograrlo si se tienen la actitud y el cariño por la organización y su gente.
Para este ejercicio de reflexión se consultaron diversos textos sobre liderazgo; especialmente, de gurús del liderazgo, tomando como referencia las características, los atributos y los hábitos que, según ellos, debe poseer una persona para ser reconocida como líder.

En el mundo de hoy, donde los valores se practican en un mínimo grado, en una sociedad influenciada por la tecnología y la globalización, sería importante contar con un líder que piense en el bien común, que trabaje arduamente en equipo, que valore las fortalezas de cada miembro y las potencialice para contrarrestar las debilidades que se presenten en el mismo equipo.

\footnotetext{
${ }^{1}$ Magíster en administración de empresas y liderazgo estratégico. Especialista en pedagogía para el desarrollo del aprendizaje autónomo. Administradora de Empresas. Docente de la Universidad Nacional Abierta y a Distancia (UNAD), con funciones de coordinadora nacional de la Maestría en Administración de Organizaciones. E-mail: martha.guerrero@unad.edu.co Grupo de Investigación ÁGORA.
} 
En el ambiente organizacional el liderazgo influye directamente en el comportamiento de la gente, y, por lo tanto, repercute en su productividad; ya no estamos en el tipo de organización donde el jefe manda y los demás obedecen: el rol del directivo debe ir más allá de dirigir y coordinar los procesos organizacionales, está obligado a motivar a su gente hacia el logro de los objetivos organizacionales e individuales. En ese sentido los directivos deben contar con unas características y cualidades especiales que les permitan lograr una influencia positiva en su equipo, desprenderse de los paradigmas del jefe y ser vistos como líderes.

Para Bennis (1994) $)^{3}$, entre los ingredientes que debe tener el líder se encuentra la integridad: "su integridad deriva del conocimiento de sí mismo, franqueza y madurez. Conoce sus fuerzas y sus debilidades, actúa de acuerdo con sus principios, y ha aprendido por experiencia como aprender y trabajar con los demás".

Es así como el líder debe conocerse a sí mismo; muy difícilmente un jefe que no sea consciente de sus debilidades y de sus fortalezas estará en capacidad de detectar las de su equipo de trabajo. Tener un pleno conocimiento de sí mismo le permite a un jefe potencializar sus habilidades hacia la dirección y el apoyo a sus colaboradores, y hacia el logro de los objetivos organizacionales.

Según Gardner (1990)4, citado por Boyett (2004), el líder debe tener habilidad en el trato con las personas, capacidad para ganarse la confianza de la gente, motivarla e impulsarla a actuar y a conseguir.

Además de lo anterior, las relaciones interpersonales son un aspecto fundamental entre el líder y su influencia en su gente; así lo considera Karl Lipper, presidente de Bavaria ${ }^{5}$, citado por Rodríguez (2008), y quien trata a sus subalternos de manera muy descomplicada, como si fueran colegas y no su jefe. Debe ser tal su grado de persuasión que pueda convertir a su grupo de trabajo en un equipo donde él es el primer integrante, y donde pueda influir en la toma de decisiones y en las acciones para seguir por parte de cada integrante.

En efecto, la motivación como elemento fundamental que debe poseer el director, se convierte en una característica esencial en su actuar como líder. "Un equipo desmotivado pasa a ser grupo y un grupo desmotivado pasa a ser una banda. No hay nexos emocionales entre las personas, entre los componentes, por tanto caen en el desánimo" (Ruiz, 2010). Es papel del líder apoyar, dar seguimiento a su equipo, hacer sentir a sus miembros que no están solos, animarlos en los momentos difíciles y elogiar sus triunfos.

Consecuentemente, la motivación influye notoriamente en generar un clima de trabajo de tipo participativo.
Frente a este tema Brunet (2011) afirma que "las fuerzas motivacionales en una organización originan actitudes generalmente favorables y la mayor parte de los empleados se sienten responsables de lo que hacen".

Por su parte, Fred. E. Fiedler, uno de los investigadores de la Teoría de la Contingencia del Liderazgo, citado por Cardona (2008), expresa que "el líder debe adecuarse a las situaciones, la eficacia del líder depende del grado en que su estilo se ajuste al contexto".

Por consiguiente, y teniendo en cuenta que los grupos de personas y las condiciones son diferentes, el líder debe actuar con un estilo de liderazgo según la situación. Para la efectividad en el estilo de liderazgo asumido es importante realizar el análisis de cada situación y determinar las acciones más apropiadas por desarrollar.

En el mismo sentido, la teoría de la contingencia encaja en lo que se conoce como liderazgo situacional, teoría del liderazgo cuyos exponentes son Paul Hersey y Kenneth Blanchard $(1996)^{6}$, citados por Zayas (2006). Ellos argumentan que el liderazgo exitoso se logra seleccionando el estilo adecuado, el cual depende del grado de disposición de los seguidores. En ese orden de ideas, un líder podrá ser participativo, persuasivo, delegador, o instructor, dependiendo de la situación y de su equipo de personas.

Por otra parte, el líder debe tener la capacidad de adaptarse a los continuos cambios. Según Jacobs $(1996)^{7}$, citado por Boyett (2004), "la capacidad de cambio de una organización es un factor clave para su buen funcionamiento tanto en el corto como en el largo plazo, las organizaciones que tendrán más éxito en el futuro serán aquellas que sean capaces de adoptar rápida y eficazmente cambios fundamentales, duraderos y de todos los sistemas".

Así pues, tal como lo plantea Bauer (2009), "El líder empresarial debe ser el primer promotor del cambio dentro de la empresa, debe ejercer cierto papel de trasgresor, de modificador de reglas".

Según lo expuesto, para poder enfrentar y afrontar el dinamismo de las variables del entorno los líderes deben estar actualizando y fortaleciendo de manera constante su desempeño profesional ${ }^{8}$. También deben desarrollar estrategias personales y estar obsesionados por la metas; deben tener una misión y proyectarse con su equipo ${ }^{9}$. Según Burt Namus, citado por Boyett (2004), una megahabilidad del liderazgo es tener una visión de futuro; es decir, mantener la vista firme en el horizonte lejano, incluso cuando se camina hacia él.

Además, según Llanes (2009), "un buen directivo debe en primer lugar, nutrirse de todos los elementos necesarios que le permitan una adecuada preparación profesional, debe dominar y conocer adecuadamente los 
diferentes métodos y estilos de dirección de acuerdo a las normas y la práctica actual toda vez que la dirección, es además de una ciencia, un arte, pues la dirección de grupos humanos requiere de una improvisación constante y actuar permanente".

Otro factor importante que hace destacar y ser reconocido al directivo como líder, por parte de su equipo de colaboradores, es que actúe según valores y códigos de ética, lo cual genera una conducta intachable y da credibilidad en sus seguidores. James MC Gregor Burns, citado por Mora (2009), argumenta que el líder que pasa por alto los componentes morales del liderazgo pasará a la historia como un malandrín o algo peor.

Cabe mencionar también que el directivo debe ser consciente de la concepción actual de liderazgo. Acerca de este tema, Galiano (2009) menciona que "el concepto moderno de Liderazgo es el de creación en las personas de un compromiso interno a objetivos sociales, de transformar el interés egoísta de las personas en una preocupación social de mayor escala". Por su parte, Martínez (2010) establece que "el liderazgo socialmente responsable busca asociar la creación de valor económico con la gestión ética, el compromiso social y el desarrollo sostenible".

De la misma manera, el líder debe actuar con valores, respetar a su gente, identificar las fortalezas de los integrantes del equipo y aprovecharlas para contrarrestar las debilidades; debe trabajar de forma continua para lograr un liderato adecuado con el cual dirija, instruya, apoye y delegue actividades a su equipo; debe estar en continuo acercamiento a su gente, $y$, como lo narran Ken Blanchard y Sheldon Bowlesel (2004) en el libro A la carga, tener el espíritu de la ardilla (o sea, fijar metas de logros y de valores), el estilo del castor (autocontrol, valorar el trabajo de su equipo y controlar las reglas) $y$, finalmente, el don del ganso (para apoyar y motivar continuamente a su equipo).

Para Pazmiño (2010), "Liderar no es simplemente, dar órdenes, aunque se den con mucha energía y con ademanes autoritarios y tampoco tiene que ver con doblegar voluntades". Frente a esta situación, y gracias a los niveles de jerarquía, en una organización las órdenes se cumplen; sin embargo, la verdadera esencia del liderazgo es ser persuasivo, y no impositivo; he ahí una gran diferencia entre el directivo y el líder.

Pero la gestión del líder debe estar apoyada por varios elementos. Thomas F. Gilbert ${ }^{10}$, citado por Boyett (2004), determina que para la efectividad organizacional deben existir unos elementos, como información, instrumentos, incentivos, conocimiento, capacidad y motivos. Con las cualidades del líder y los elementos organizacionales se puede pensar en un liderazgo efectivo.
En esa misma línea, el directivo debe actuar según paradigmas contemporáneos del liderazgo: la acción, la innovación, el trabajo en equipo, la inteligencia emocional, la gestión del cambio y la gestión de la excelencia.

Para Jack Welch (2006) ${ }^{11}$, apoyado en su prolongada experiencia como líder, existen ocho reglas para ejercer el liderazgo:

1. Los líderes consiguen que su equipo mejore continuamente: aprovechan cualquier encuentro para evaluar, aleccionar y otorgar confianza a sus empleados.

2. Los líderes no solo se aseguran de que el personal entienda la visión de la empresa, sino también, de que la viva y la respire.

3. Los líderes se meten en la piel de su personal e irradian energía positiva y optimismo.

4. Los líderes establecen la confianza mediante la sinceridad, la transparencia y el honor.

5. Los líderes tienen valor para tomar decisiones impopulares y confían en su instinto.

6. Los líderes cuestionan insistentemente todo, con una curiosidad que raya en el escepticismo, para asegurarse de que sus preguntas tienen unas respuestas plausibles.

7. Los líderes inspiran con su ejemplo la toma de decisiones arriesgadas y el aprendizaje continuado.

8. Por último, un líder siempre celebra los triunfos. El liderazgo es un desafío donde se entrelazan equilibrios, responsabilidades y presión.

Las cualidades anotadas en este escrito deben llevar al directivo a reflexionar sobre qué es ser líder.

Ser líder es poseer unas características especiales que faciliten la interacción con el equipo de trabajo y logren un alto grado de influencia y de persuasión hacia ese grupo, de tal forma que lo sigan voluntariamente, confiando en su dirección y su asesoría.

Ser líder es ser exitoso, es poseer habilidades y cualidades que lleven a la persona a lograr sus objetivos y los de su equipo.

Ser líder es ser capaz de analizar las situaciones y tomar la decisión adecuada para solucionar problemas; es asumir con modestia el éxito y aceptar los fracasos sin sentirse derrotado; es tener una permanente disposición para actuar, pensando siempre en lograr lo que se propone.

Ser líder es hallarse en constante percepción de todo lo que sucede a su alrededor; es tener la habilidad de trabajar coordinadamente en equipo; es entender que el trabajo en equipo está basado en las personas, y no en las actividades; es ser asertivo y tener seguridad en lo que dice y en lo que hace. 
Ser líder es ganarse la confianza del otro; es crear un ambiente que le permita a su grupo aprender, crecer y superarse para alcanzar la excelencia.

Ser líder es actuar con ética y valores; es tener autoridad moral para actuar, dar ejemplo y dirigir.

Finalmente, para ser líder es indispensable sentirse vivo, poder disfrutar lo que se tiene, luchar por conseguir lo que se desea y lograr los objetivos sin el perjuicio de nadie.

\section{Conclusiones}

Las organizaciones necesitan a directivos que sean líderes, que sean estrategas, que tengan una misión clara y estén obsesionados por cumplir con las metas, que estén en contacto continuo con la gente y actúen según principios y valores.

El poder que ejercen los directivos gracias a su posición jerárquica en la organización genera una percepción negativa del clima, en tanto que un directivo con liderazgo genera satisfacción en los colaboradores y sentido de pertenencia hacia la organización.

Pese a lo anterior, ser líder no es fácil: exige habilidades especiales, pero si la persona lo siente y lo desea, si tiene compromiso social y amor por su organización, por su profesión y por lo que hace, podrá liderar un equipo de trabajo, y lograr los objetivos particulares y los organizacionales. Además, tal como lo afirma Stephen R. Covey, citado por Boyett (2004), "El líder total se convierte en un servidor, en alguien que es un recurso, en alguien que facilita las cosas.

Entonces, hay que trabajar para ello con dedicación, compromiso, mejoramiento personal $y$, sobre todo, con las ganas de hacerlo y de lograrlo; así, las organizaciones podrán contar con directivos que sean verdaderos líderes.

\section{Referencias bibliográficas}

Barker, J. A. (1995). Paradigmas. El negocio de descubrir el futuro. Bogotá: McGraw Hill.

Bennis,W., Nanus, B. (1994). Líderes, las cuatro calves del liderazgo eficaz. Bogotá: Editorial Norma.

Blanchard, K. \& Bowles, S. (2004). A la Carga, cómo aprovechar al máximo el potencial de las personas en su empresa. Bogotá: Grupo Editorial Norma.

Boyett, J. \& Boyett, J. (2004). Lo mejor de los gurús. Barcelona: Ediciones Gestiòn 2000 S. A.

Brunet, L. (2011). El clima de trabajo en las organizaciones. México: Editorial Trillas.

Cardona, P. (2008). Cómo desarrollar las competencias de liderazgo (3a. ed.). Navarra-España: Editorial EUNSA.
Galiano, G., Jesús M. (2009). Algunas reflexiones acerca de liderazgo. Santa Fe, Argentina: Editorial El Cid Editor.

Hiebaum de Bauer, K. (2009). Mi desarrollo como un líder basado en mando y conceptos de comportamiento organizativo. Buenos Aires: Editorial El Cid Editor.

Llanes Belett, R. (2009). Un estilo de dirección adecuado y el liderazgo correcto de los directivos actuales, un medio importante de lograr una mayor eficiencia empresarial. Buenos Aires: Editorial El Cid Editor.

Martínez Herrera, H. (2010). Liderazgo responsable. Bogotá: Ecoe Ediciones.

Mora Vanegas, C. (2009). El liderazgo proactivo en la empresa moderna. Buenos Aires: Editorial El Cid Editor.

Pazmiño Cruzatti, I. (2010). Liderazgo, más que una estrategia gerencial. Quito: EDITEKA Ediciones.

Robbins, S. \& Coulter, M. (2005). Administración. México: Pearson Educación.

Rodríguez Múnera, M. (2008). Perfiles empresariales. Bogotá: Casa editorial El Tiempo.

Ruiz, M. (2010). ¿Para qué sirve un líder?: lecciones de liderazgo aplicadas a la empresa, la política y la vida. Madrid: Ediciones Díaz de Santos.

Welch, J. (2006). Ganar. México: Editorial Collins.

Zayas Agüero, P. M. \& Cabrera Ferreiro, N. (2006). Liderazgo empresarial. Cuba: Universidad De Holguín "Oscar Lucero Moya".

\section{Notas}

2 Según el Diccionario de la Real Academia Española, "coach. Voz inglesa que significa 'persona que prepara o adiestra a otra en algo, especialmente en la práctica de un deporte'. Su uso es innecesario en español, por existir términos como entrenador y preparador, de sentido equivalente".

${ }^{3}$ Bennis es profesor de Administración de Empresas en la Universidad de Southern California. Es también autor de muchos trabajos sobre el liderazgo.

${ }^{4}$ John W. Gardener fue vicepresidente y presidente de la Fundación Carnegie para el Progreso de la Enseñanza de Ios EE.UU.; en la actualidad es profesor de asesoría de empresa en la Escuela Superior de Negocios de la Universidad de Stanford, y presidente de la Liga Nacional Cívica.

${ }^{5}$ Bavaria es una de las empresas privadas más grandes de Colombia. ${ }^{6}$ Paul Hersey es profesor distinguido de los estudios de dirección de la Universidad del Sudeste de la Nova, de la Universidad del Norte de Illinois, de la Universidad del estado de California, de la Universidad de Arkansas, y de la Universidad de Ohio. Kenneth Blanchard es doctor en administración de la educación y liderazgo; además, es un prominente conferencista, consultor y escritor.

7 Robert Jacobs es profesor de gestión de operaciones de la Universidad de Indiana.

8 Sobre este tema José Darío Uribe, gerente del Banco de la 
Republica, afirma que dedica el $25 \%$ del tiempo a investigar, asiste por lo menos a 2 seminarios internacionales sobre su campo de actuación cada año, y debate con colegas sobre temas concretos y mejores prácticas, para luego aplicarlas a su propia organización.

${ }^{9}$ La visión de futuro es una de las siete megahabilidades que plantea Burt Namus.
${ }^{10}$ Thomas F. Gilbert ha sido aclamado como el padre fundador de la tecnología de la efectividad.

11 Jack Welch fue directivo de la General Electric, empresa a la que salvó de la quiebra. En su libro Ganar detalla las ocho reglas para ejercer el liderazgo. 